\title{
Perceived subject outcomes and impact on health-related quality of life associated with diet using the new Food Benefits Assessment $\left(\mathrm{FBA}^{\mathrm{O}}\right)$ questionnaire: development and psychometric validation
}

\author{
Denis Guyonnet ${ }^{1, *}$, Olivier Chassany ${ }^{2}$, Céline Picard ${ }^{1}$, Isabelle Guillemin ${ }^{3}$, Juliette Meunier ${ }^{3}$, \\ Eva Seignobos ${ }^{3}$, Marc Vigneux $^{3}$, Taous Lassel ${ }^{1}$ and Elyse Trudeau ${ }^{3}$ \\ ${ }^{1}$ Danone Research, RD 128, F-91767 Palaiseau Cedex, France: ${ }^{2}$ Clinical Research Department, Assistance \\ Publique Hôpitaux de Paris \& Denis-Diderot University, Paris, France: ${ }^{3}$ Mapi Values, Lyon, France
}

Submitted 27 April 2007: Accepted 24 November 2007: First published online 18 February 2008

\begin{abstract}
Objective: To assess the perceived outcomes associated with diet/food intake in the general adult population.

Design and subjects: The Food Benefits Assessment $\left(\mathrm{FBA}^{(}\right)$questionnaire was developed from subjects' verbatim transcripts ( $n$ 18) and after comprehension tests ( $n$ 5). Normal-weight ( $n$ 130) and overweight ( $n$ 67) subjects then completed the final questionnaire twice, $7 \mathrm{~d}$ apart. Psychometric properties were assessed, including construct validity by principal components analysis (PCA), concurrent validity (Spearman coefficient) with the Short Form-36 scale (SF-36), knowngroup validity by comparing FBA dimension scores according to lifestyle and clinical variables, internal consistency reliability (Cronbach's $\alpha$ ) and test-retest reproducibility in stable subjects over 1 week (intraclass correlation coefficient, ICC). Results: PCA and Multitrait analysis confirmed the final version of the FBA comprising forty-one items split into seven dimensions (vitality; digestive comfort; physical appearance; well-being; snacking; disease prevention; aesthetics). All dimensions displayed good item convergent validity $(0 \cdot 44$ to $0 \cdot 80)$, good concurrent validity (highest correlation between well-being dimension of FBA and mental health scale of SF-36, $r=0 \cdot 83$ ) and good known-group validity and reproducibility (ICC $\geq 0 \cdot 76$ ); internal consistency reliability was good to excellent (Cronbach's $\alpha=0 \cdot 79$ to $0 \cdot 91$ ).

Conclusion: The FBA is the first valid and reliable questionnaire that allows the assessment of diet effects and impact as perceived by subjects. It is a good candidate in the nutrition field for further use in specific population settings and with a particular food or daily diet. Linguistically validated English (UK and US) and German versions of the questionnaire are available.
\end{abstract}

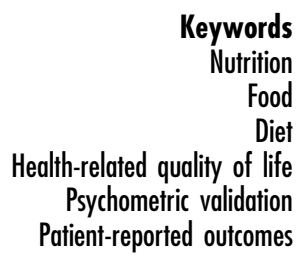

Patient-reported outcomes such as health-related quality of life (HRQOL) are becoming increasingly necessary and relevant in the field of nutrition. It is now well established that nutrition influences outcomes; for example, in patients with irritable bowel syndrome (IBS), chronic constipation and faecal incontinence, diabetes and cancer $^{(1-5)}$. However, HRQoL questionnaires are seldom used in the nutrition field. The measurement instruments that are being used are either not specific to nutrition, which is the case for the generic Short Form-36 (SF-36) questionnaire, or are specific to a population setting, such as the Impact of Weight on Quality of Life and its French version (the Quality of Life, Obesity and Dietetics rating scale), assessing HRQoL in obese patients ${ }^{(6-8)}$. FFQ are used to provide estimates of dietary intake over time in one specific population or to identify people with high intake of dietary fat and/or low intake of fibre, fruits and vegetables $^{(9,10)}$. The Mini Nutritional Assessment is used to quickly screen elderly persons at risk of malnutrition $^{(11,12)}$. The Seniors in the Community Risk Evaluation for Eating and Nutrition scale was developed to determine the link between nutritional risk and HRQoL, and tested in frail older adults ${ }^{(13)}$. Numerous other questionnaires are available that are not directly related to nutrition but on which nutrition may have a considerable impact, such as questionnaires developed for digestive pathologies and functional digestive disorders. Among these, the Irritable Bowel Syndrome Quality of Life 
measures quality of life in IBS patients and the Gastrointestinal Quality of Life Index questionnaire assesses the impact of anal incontinence and chronic constipation on HRQOL ${ }^{(14,15)}$. To our knowledge, no questionnaire is available that assesses the effects of food, irrespective of diet, on HRQOL in the general adult population.

The importance of HRQOL and patient-reported outcomes as meaningful and valuable clinical end points has been increasing over recent years. They are now recommended by the US Food and Drug Administration (FDA) and the European Agency for the Evaluation of the Medicinal Products (EMEA) in order to obtain a claim, to differentiate a product or to communicate about a product. This therefore requires reliable and validated tools developed following a rigorous methodology ${ }^{(16-18)}$.

The current manuscript presents the development of the Food Benefits Assessment $\left(\mathrm{FBA}^{\mathbb{C}}\right.$ ) questionnaire that evaluates the perceived positive and negative outcomes associated with diet/food intake in the general adult population. This instrument might help physicians who still face difficulties when having to advise and prescribe a modified diet to subjects ${ }^{(19)}$. The development methodology and psychometric validation of the French FBA questionnaire are further described, as well as the English (UK and US) and German linguistic validations. People's awareness of the effects of a healthy diet and the identification and definition of these effects are discussed together with their consequences on HRQOL.

\section{Materials and methods}

\section{Development of the Food Benefits Assessment questionnaire}

Based on a thorough literature review on people's concerns regarding food and daily diets, interview guides were elaborated by a scientific committee comprising nutrition specialists, methodologists and health psychologists. Interview guides explored subjects' opinions on food/diet, satisfaction in terms of pleasure and expectations regarding food, vitality, sleep, digestive symptoms, satiety, psychology and projection in the future. A total of eighteen adults aged 18 to 65 years, with no aversion to dairy products, were recruited from the general population by three general practitioners. In order to identify subjects' representations of a good/bad daily diet, semistructured face-to-face interviews with ten of the above adults were first conducted by a health psychologist. They resulted in the establishment of a conceptual model, which was validated by subsequent structured face-toface interviews performed with the remaining eight adults. During a scientific committee meeting, subjects' verbatim transcripts were analysed and contributed to the amendment and refinement of the initial conceptual model and to item elicitation. In order to confirm its clarity, comprehensiveness and cultural acceptability, the questionnaire was tested for comprehension among another five adult subjects recruited by general practitioners using the same inclusion criteria. During these interviews, subjects were asked to complete the questionnaire, to answer questions in order to evaluate their understanding, interpretation and the pertinence of each item, and to make suggestions.

\section{Linguistic validation of the Food Benefits Assessment questionnaire}

The final questionnaire was translated into English (UK and US) and German using standard linguistic translation methodology ${ }^{(20)}$, including forward then backward translation. A comprehension test was then performed with subjects from the target country in order to assess the clarity, appropriateness of wording and acceptability of the translated questionnaire. After proofreading steps, the translated questionnaire was linguistically validated.

\section{Study design and populations}

The study, conducted in France, was open and observational. Each of the thirty-four general practitioners participating in the study recruited six adults, including four normal-weight subjects and two overweight subjects. Inclusion criteria were as follows: subjects had to be between 20 and 65 years old, with BMI of less than $25 \mathrm{~kg} / \mathrm{m}^{2}$ for the normal-weight group and BMI between 25 and $30 \mathrm{~kg} / \mathrm{m}^{2}$ for the overweight group. Subjects were not included if they had a threatening disease that could have affected their short-term vital prognosis, food allergies, followed restrictive diets (e.g. low-sodium, low-protein), showed signs of eating disorders (e.g. anorexia nervosa, bulimia nervosa, binge eating disorder) or were participating in a clinical trial; women were not to be pregnant. These criteria were assessed in the case report form completed by the general practitioners at the inclusion visit (day 0). On the same day, subjects were asked to complete the FBA and the generic SF-36 questionnaires ${ }^{(21)}$. Seven days later (day 7), they were asked to fill out the FBA questionnaire and five questions assessing possible changes (diet, hospitalisation, start of a diet, change in weight, interfering event in subjects' life) during this period of time.

FBA finalisation analysis was performed with the 'finalisation set' population, i.e. subjects who had completed at least one FBA item at day 0. Psychometric properties were determined on the cross-sectional population, defined as subjects who met the inclusion criteria and whose questionnaire was exploitable (i.e. less than $50 \%$ of missing data). Test-retest analyses were performed on stable subjects between day 0 and day 7 visits and for whom the FBA questionnaire was assessable (less than $50 \%$ of missing data). Subjects who answered 'no' to all five additional questions they were asked at day 7 were considered as stable. 


\section{Statistical analyses}

Principal components analysis (PCA) using Varimax rotation was used to ascertain the dimensions of the FBA questionnaire. Eigenvalues greater than 1 were fixed as defining a factor. The final structure of the FBA was evaluated by performing a Multitrait analysis (MA) describing item convergent and item discriminant validity. The correlation between each item and its own scale is satisfactory if it achieves $\geq 0 \cdot 40^{(22)}$. Item discriminant validity requires that each of the items shares a higher correlation with its own scale than with other scales. Floor and ceiling effects were determined in order to check that there was no bias related to a high percentage of subjects having the lowest or the highest possible score, respectively. FBA scale-scale correlations (Spearman coefficients) were calculated. Concurrent validity was investigated by analysing correlations (Spearman coefficients) between FBA and SF-36 scores, as well as mental component score (MCS) and physical component score (PCS) ${ }^{(21)}$. The SF-36 scores and MCS and PCS summary scores were generated following the standard instructions provided in the SF-36 Health Survey Manual and Instruction Guide ${ }^{(21)}$. Known-group validity evaluates the extent to which the questionnaire is able to discriminate between groups of subjects according to sociodemographic and lifestyle criteria. Known-group validity can be evaluated when logical relationships are expected for groups of patients known or thought to differ on relevant variables ${ }^{(16)}$. It is established through the development of hypotheses about the behaviour of scores of the HRQOL instrument in various situations. In the present study, weight, snacking between meals and self-rated general health (SF-36) were the criteria evaluated. Kruskal-Wallis (when comparing three groups or more of patients) and Mann-Whitney Wilcoxon (when comparing two groups of patients) non-parametric tests were performed. Internal consistency reliability of the FBA was assessed by determination of Cronbach's $\alpha$ coefficient: a value of $0 \cdot 70$ or above was considered satisfactory. Reproducibility of a questionnaire refers to its stability over time, which was measured by the intraclass correlation coefficient (ICC) between day 0 and day 7 .

The threshold for statistical significance was fixed at 5\%. All data processing and analyses were performed using the Statistical Analysis Systems statistical software package version 9 (SAS Institute, Cary, NC, USA).

\section{Results}

\section{Questionnaire development}

Exploratory interviews ( $n$ 10) conducted in France with the general adult population following interview guides resulted in the identification of six major general concepts: sleep, symptoms, psychological aspects, vitality, aesthetics and satisfaction. The subsequent structured interviews with another eight subjects allowed the definition of more detailed domains related to symptoms (satiety and gastrointestinal symptoms), vitality (energy, intellectual, physical fatigue/attention), well-being, aesthetics (weight, self-esteem, physical appearance), sleep, psychological aspects (stress, anxiety, well-being) and satisfaction (pleasure, self-satisfaction, food satisfaction, conviviality, perception of the results) (Fig. 1). Sixty-nine items covering these domains were formulated from subjects' verbatim transcripts. Following comprehension testing with five subjects, eight items were deleted as they were not relevant; the wording of other items was modified because these were not clear for the subjects. The resulting initial version of the FBA questionnaire consisted of sixty-one items related to gastrointestinal and digestive comfort symptoms (fourteen items), satiety (four), vitality (eleven), well-being (ten), aesthetics (eight), sleep (three), disease prevention (four), projection in the future (four), pleasure (two) and satisfaction (one).

\section{Study design and population characteristics}

All 204 FBA questionnaires received at day 0 were exploitable. Among the 172 questionnaires received at day 7 , only three were not exploitable due to too many missing items. Of the 204 subjects, seven (3\%) were removed as they did not meet the inclusion criteria. Sociodemographic and clinical characteristics of the cross-sectional population are summarised in Table 1.

\section{Acceptability of the Food Benefits Assessment questionnaire}

Ninety-three per cent of the cross-sectional population had no missing data at day 0 and $94 \%$ at day 7 . The mean percentage of missing items per subject was $0.45 \%$ at day 0 and $2 \cdot 41 \%$ at day 7 .

\section{Factorial structure of the Food Benefits Assessment questionnaire and scoring}

In order to hypothesise the number of dimensions within the FBA and to set up a scoring algorithm, a series of analyses comprising PCA with Varimax rotation followed by MA were first performed on the initial pilot version (ten domains and sixty-one items) with the 'finalisation set' population ( $n$ 197). These successive analyses yielded eight factors with eigenvalues greater than 1. Eighteen items were deleted because they were strongly correlated with another item, did not meet the convergent and discriminant validity criteria or displayed a floor effect. The remaining forty-three items covered the dimensions of vitality, digestive comfort, disease prevention, well-being, aesthetics, physical appearance, snacking and pleasure.

The version was further tested on the cross-sectional population ( $n$ 197). All items met the item convergent and discriminant validity criteria. All dimensions had satisfactory internal consistency reliability, with Cronbach's $\alpha$ 


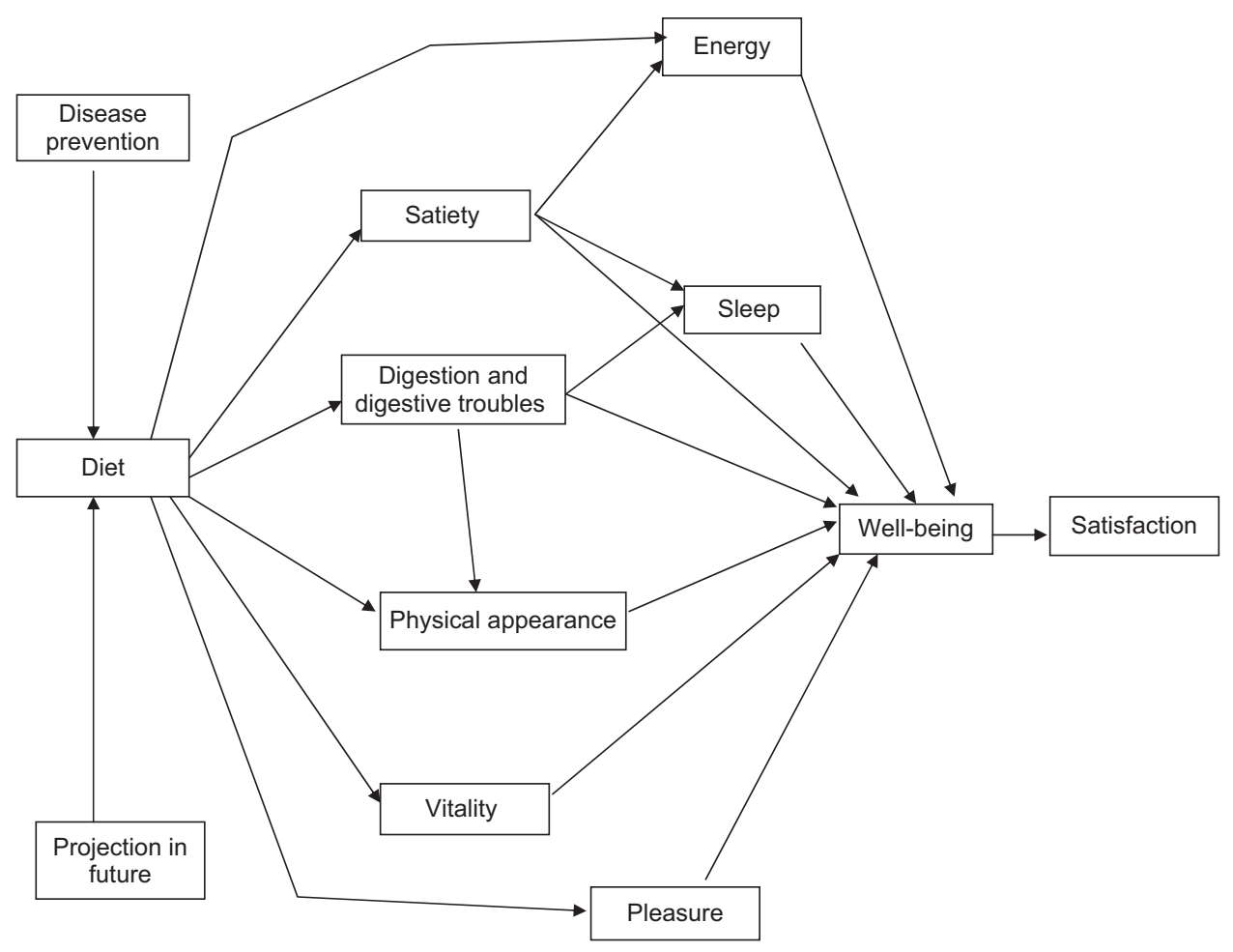

Fig. 1 Conceptual model of the Food Benefits Assessment questionnaire

ranging from $0 \cdot 75$ to 0.91 (Table 2 ). The reproducibility of all dimensions was good, except for the pleasure dimension that had an ICC value below the recommended threshold (ICC $=0.53$ ) (Table 2). Because of its low reproducibility and its weak known-group validity, together with its low number of items, the pleasure dimension was deleted from the final version of the FBA questionnaire.

PCA with Varimax-rotated factor pattern with the number of factors forced to seven confirmed the sevendimension structure of the FBA, accounting for $64 \cdot 7 \%$ of the total variance.

The subsequent MA showed that item-scale correlation was good for all the items within their respective dimension, ranging from $0 \cdot 44$ to $0 \cdot 80$. Satisfactory item discriminant validity was reached for all items with the exception of one about the 'looking well' concept, which was common to the aesthetics and well-being dimensions.

FBA scale-scale correlations were weak, with Spearman correlation coefficients varying from 0.02 (correlation between physical appearance and disease prevention dimensions) to $0 \cdot 70$ (correlation between well-being and vitality dimensions).

Finally, no floor or ceiling effects were observed, as less than $20 \%$ of the subjects answered either of the two extreme response choices, i.e. never/certainly not or always/certainly. Results are summarised in Table 3.

Therefore, the final validated version of the FBA comprised forty-one items divided into seven dimensions that covered vitality (ten items), digestive comfort (nine items), disease prevention (six items), well-being (six items), aesthetics (five items), physical appearance (three items) and snacking (two items), as perceived by subjects. A 5-point Likert-type response scale ranging from 'certainly' to 'certainly not' or 'always' to 'never' was common to all items.

All FBA items were scored on a categorical rating scale varying from 1 to 5 , from which a mean score was calculated. The score of each of the seven dimensions was determined based on a linear transformation of the mean score, and ranged from 0 to 100. Higher scores indicated higher positive impact and/or satisfaction with the daily diet.

\section{Psychometric properties of the final Food Benefits Assessment questionnaire}

The following analyses were conducted with the 197 normal-weight and overweight subjects of the crosssectional population, except wherever specified.

A satisfactory to excellent internal consistency reliability was observed (Table 3 ).

The reproducibility of the questionnaire was assessed in subjects who reported stable food habits and health status between baseline and day 7 visits ( $n$ 120). ICC scores exceeded the 0.70 threshold for all dimensions (Table 3).

Concurrent validity of the FBA was established by comparing FBA dimensions with SF-36 sub-scales (Table 4). 
Table 1 Sociodemographic, food and lifestyle habits and clinical characteristics of the cross-sectional population at baseline $(n$ 197)

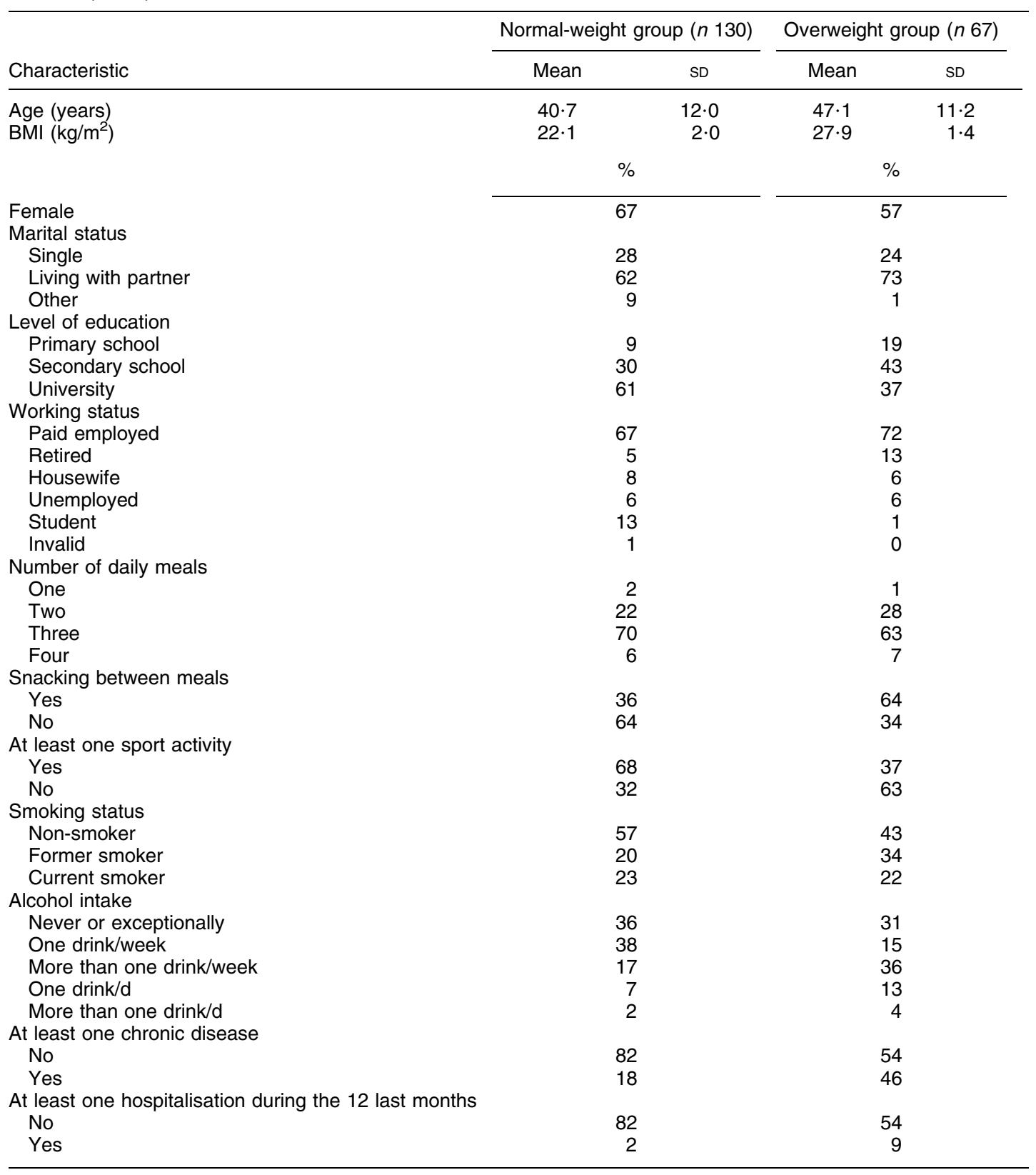

The highest significant correlation was observed between the well-being dimension of FBA and the mental health scale of SF-36 (Spearman coefficient $r=0 \cdot 83 ; P<0 \cdot 001$ ). Both vitality and well-being dimensions of FBA were strongly correlated to social functioning, mental health, vitality and the MCS of SF-36 (coefficients ranging from $0 \cdot 60$ to $0 \cdot 83 ; P<0 \cdot 001)$. Snacking and disease prevention dimensions were the least correlated to SF-36 sub-scales (coefficients ranging from $0 \cdot 02$ to $0 \cdot 27$ ).

Known-group validity of the FBA was assessed with the cross-sectional population at baseline ( $n$ 197). Overall, scores of all dimensions decreased as subjects' BMI increased, the differences between the normal-weight and overweight groups of subjects being significant $(0 \cdot 001<P \leq 0 \cdot 012)$ except for the disease prevention dimension $(P=0 \cdot 051)$ (Fig. 2$)$. The highest significant difference $(P<0 \cdot 001)$ between the two groups was observed for the physical appearance and the digestive comfort dimensions (respective score: 65 and 71 for normal-weight subjects, 41 and 60 for overweight subjects).

Regarding lifestyle variables, no significant differences were observed between groups based on the number of daily meals (data not shown). In contrast, scores of all dimensions, except disease prevention and well-being, were significantly different between subjects who had the habit of snacking between meals compared with those 
who did not; the lowest scores were obtained with subjects who were used to snacking (Fig. 3). Finally, all dimensions, except disease prevention, significantly discriminated between groups of subjects according to their general health perceptions as defined by the SF-36, with the lowest scores observed for the group of subjects with 'fair/poor' general health (Fig. 4).

\section{Discussion}

Daily diet and nutrition as a whole have become highly fashionable and popular topics for both individuals and society in general. This probably explains, at least in part,

Table 2 Internal consistency reliability and reproducibility of the Food Benefits Assessment (FBA) questionnaire with forty-three items and eight dimensions

\begin{tabular}{lccc}
\hline FBA dimension & Number of items & $\begin{array}{c}\text { Cronbach's } \alpha \\
(n \text { 188 })\end{array}$ & $\begin{array}{c}\text { ICC } \\
(n \text { 120 })\end{array}$ \\
\hline Vitality & 10 & 0.91 & 0.76 \\
Digestive comfort & 9 & 0.89 & 0.79 \\
Disease prevention & 6 & 0.88 & 0.77 \\
Well-being & 6 & 0.87 & 0.80 \\
Aesthetics & 5 & 0.82 & 0.80 \\
Physical appearance & 3 & 0.79 & 0.85 \\
Snacking & 2 & 0.81 & 0.78 \\
Pleasure & 2 & 0.75 & 0.54 \\
\hline
\end{tabular}

ICC, intraclass correlation coefficient. the very good acceptability of the FBA questionnaire by all subjects who participated in its development, especially as they were directly interviewed about the benefits/negative effects they thought to be related to their diet. Patients' outcomes are an essential and highly valuable source of information in that they constitute subjective assessment in terms of perceptions, beliefs and needs for a treatment and/or disease or particular condition. Thus, the importance of patient perspectives on disease impact and response to therapy is increasingly acknowledged, with patient-reported outcomes, including HRQOL, providing relevant information. Even though the importance of patient-reported outcomes and HRQOL outcomes concerning changes in diet has increased in the field of nutrition, questionnaires are still not commonly used in this field. The relationship between diet/food and HRQoL may seem obvious and intuitive (psychologically, with depression and eating disorders; socially, with social class and obesity). However, to our knowledge, no study is available that fully addresses this link in a general adult healthy population.

The FBA is the first questionnaire that assesses the effects of daily diet and the impacts of these effects, as directly perceived by a population of normal-weight and overweight adults. It is available in French, UK and US English and German, which will allow its wider use in international studies. A psychometric validation of these

Table 3 Floor and ceiling effects, internal consistency reliability ( $n$ 184) and reproducibility by test-retest over 1 week ( $n$ 120) of the final Food Benefits Assessment (FBA) questionnaire (forty-one items and seven dimensions)

\begin{tabular}{|c|c|c|c|c|c|}
\hline \multirow[b]{2}{*}{ FBA dimension } & \multirow[b]{2}{*}{ Floor effect (\%) } & \multirow[b]{2}{*}{ Ceiling effect (\%) } & \multirow[b]{2}{*}{ Cronbach's $\alpha$} & \multicolumn{2}{|c|}{ Reproducibility over $7 d$} \\
\hline & & & & Missing data & ICC \\
\hline Vitality & 0 & $1 \cdot 1$ & 0.91 & 2 & 0.76 \\
\hline Digestive comfort & 0 & $2 \cdot 2$ & $0 \cdot 89$ & 2 & 0.79 \\
\hline Disease prevention & 0 & $15 \cdot 8$ & $0 \cdot 88$ & 1 & 0.77 \\
\hline Well-being & 0 & $1 \cdot 1$ & $0 \cdot 87$ & 0 & 0.80 \\
\hline Aesthetics & 0 & $2 \cdot 7$ & $0 \cdot 82$ & 1 & $0 \cdot 80$ \\
\hline Physical appearance & 0.5 & $1 \cdot 6$ & $0 \cdot 79$ & 1 & 0.85 \\
\hline Snacking & $2 \cdot 7$ & $5 \cdot 4$ & $0 \cdot 81$ & 1 & 0.78 \\
\hline
\end{tabular}

ICC, intraclass correlation coefficient.

Table 4 Spearman correlation coefficients between scores on the Food Benefits Assessment (FBA) questionnaire and the Short Form-36 (SF-36) sub-scales ( $n 186$ due to missing data)

\begin{tabular}{|c|c|c|c|c|c|c|c|c|c|c|}
\hline \multirow[b]{2}{*}{ FBA dimension } & \multicolumn{10}{|c|}{ SF-36 sub-scales and component scales } \\
\hline & PF & $\mathrm{RP}$ & BP & $\mathrm{GH}$ & VT & SF & RE & $\mathrm{MH}$ & PCS & MCS \\
\hline Vitality & $0 \cdot 25^{\star \star \star}$ & $0 \cdot 43^{\star \star \star}$ & $0 \cdot 45^{\star \star \star}$ & $0 \cdot 52^{\star \star \star}$ & $0 \cdot 71^{\star \star \star}$ & $0 \cdot 60^{\star \star \star}$ & $0 \cdot 53^{\star \star \star}$ & $0 \cdot 66^{\star \star \star}$ & $0 \cdot 28^{\star \star \star}$ & $0 \cdot 68^{\star \star \star}$ \\
\hline Digestive comfort & $0 \cdot 22^{\star *}$ & $0 \cdot 39^{\star \star *}$ & $0 \cdot 40^{\star \star *}$ & $0 \cdot 37^{\star \star *}$ & $0 \cdot 45^{\star \star \star}$ & $0 \cdot 49^{\star \star \star}$ & $0 \cdot 40^{\star \star \star}$ & $0 \cdot 45^{\star \star \star}$ & $0 \cdot 29^{\star \star \star}$ & $0 \cdot 48^{\star \star \star}$ \\
\hline Disease prevention & $0 \cdot 10^{\mathrm{NS}}$ & $0.08^{\mathrm{NS}}$ & $0.04^{\mathrm{NS}}$ & $0 \cdot 11^{\mathrm{NS}}$ & $0.03^{\mathrm{NS}}$ & $-0.07^{N S}$ & $0.06^{\mathrm{NS}}$ & $0.02^{\mathrm{NS}}$ & $0 \cdot 11^{\mathrm{NS}}$ & $-0.02^{N S}$ \\
\hline Well-being & $0 \cdot 29^{\star \star \star}$ & $0 \cdot 36^{\star \star \star}$ & $0 \cdot 48^{\star \star \star}$ & $0 \cdot 45^{\star \star \star}$ & $0 \cdot 71^{\star \star \star}$ & $0 \cdot 70^{\star \star \star}$ & $0 \cdot 51^{\star \star \star}$ & $0 \cdot 83^{\star \star \star}$ & $0 \cdot 22^{\star \star}$ & $0.79^{\star \star \star}$ \\
\hline Aesthetics & $0 \cdot 22^{* \star}$ & $0 \cdot 26^{\star \star *}$ & $0 \cdot 38^{\star \star \star}$ & $0 \cdot 53^{\star \star \star}$ & $0.48^{\star \star \star}$ & $0 \cdot 40^{\star \star *}$ & $0 \cdot 26^{\star \star \star}$ & $0 \cdot 50^{\star \star \star}$ & $0 \cdot 28^{\star \star \star}$ & $0 \cdot 45^{\star \star \star}$ \\
\hline Physical appearance & $0 \cdot 35^{\star \star \star}$ & $0 \cdot 31^{\star \star \star}$ & $0 \cdot 41^{\star \star \star}$ & $0 \cdot 43^{\star \star \star}$ & $0 \cdot 44^{\star \star \star}$ & $0 \cdot 43^{\star \star \star}$ & $0 \cdot 28^{\star \star \star}$ & $0 \cdot 47^{\star \star \star}$ & $0 \cdot 34^{\star \star \star}$ & $0 \cdot 42^{\star \star \star}$ \\
\hline Snacking & $0.08^{N S}$ & $0.05^{N S}$ & $0 \cdot 14^{\star}$ & $0 \cdot 21^{\star *}$ & $0 \cdot 27^{\star \star \star}$ & $0 \cdot 18^{\star}$ & $0.04^{\mathrm{NS}}$ & $0 \cdot 22^{\star *}$ & $0 \cdot 12^{N S}$ & $0 \cdot 19^{\star *}$ \\
\hline
\end{tabular}

PF, Physical Function; RP, Role Physical; BP, Bodily Pain; GH, General Health; VT, Vitality; SF, Social Functioning; RE, Role Emotional; MH, Mental Health; PCS, Physical Component Scale; MCS, Mental Component Scale.

Correlations were statistically significant: ${ }^{\star} P \leq 0.05,{ }^{* \star} P \leq 0.01,{ }^{* \star} P<0.001$. 


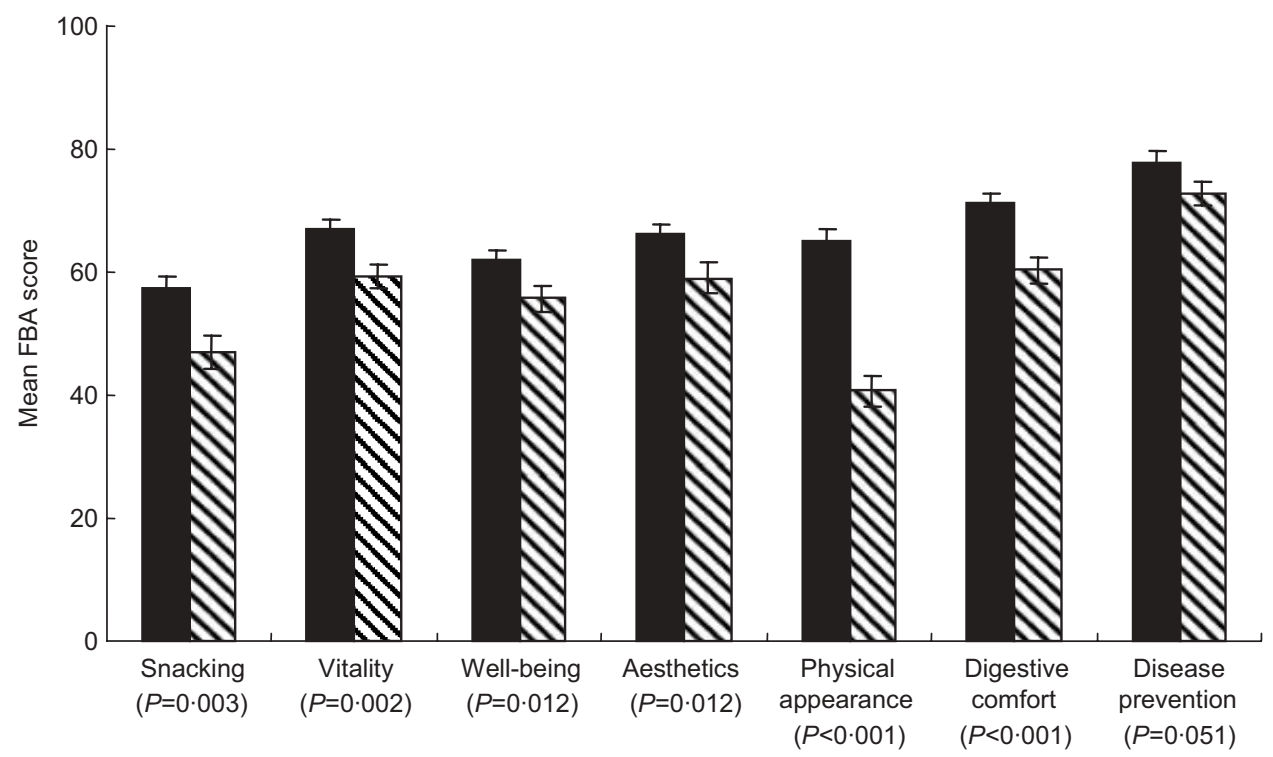

Fig. 2 Comparison between scores of different dimensions of the Food Benefits Assessment (FBA) questionnaire according to BMI groups: $\mathbf{B M I}<25 \mathrm{~kg} / \mathrm{m}^{2}(n 130) ; \mathbf{S}, \mathrm{BMI} \geq 25 \mathrm{~kg} / \mathrm{m}^{2}(n 67)$. Values are means with their standard deviation represented by vertical bars

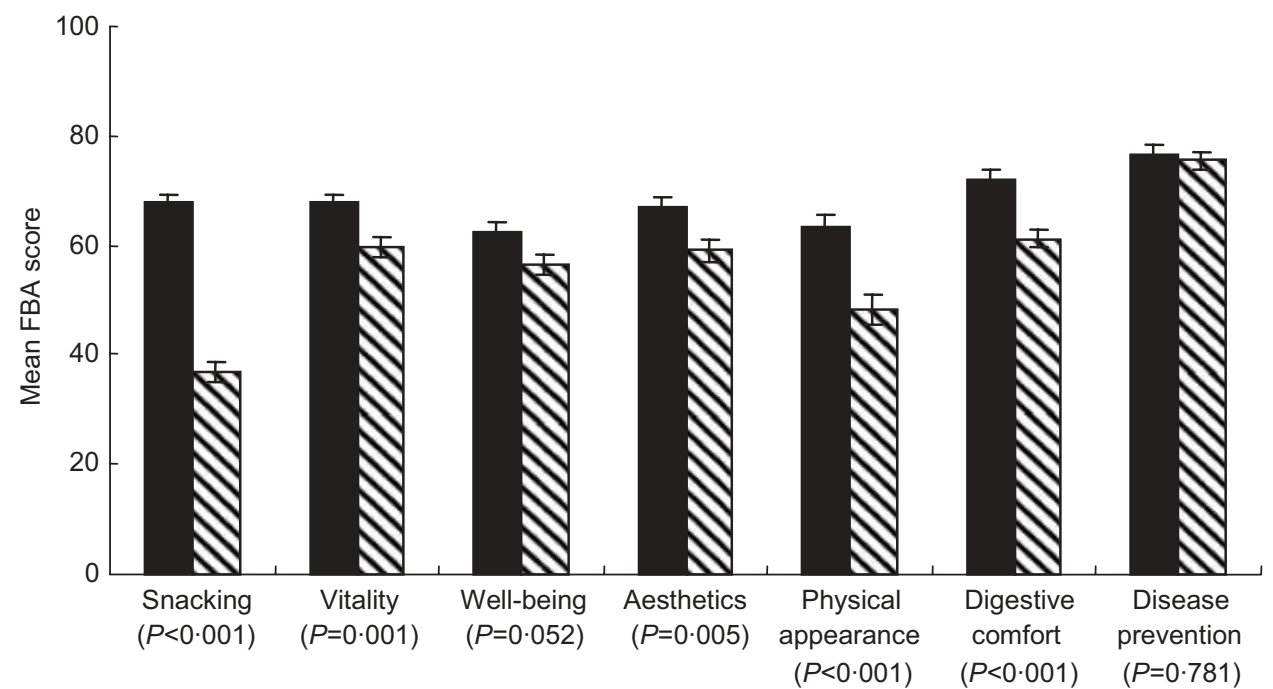

Fig. 3 Comparison between scores of different dimensions of the Food Benefits Assessment (FBA) questionnaire according to subjects' behaviour regarding snacking: $\mathbf{\square}$, no snacking between meals ( $n$ 106); $\mathbf{\Sigma}$, snacking between meals ( $n$ 90). Values are means with their standard deviation represented by vertical bars

versions will be required before their use in further studies. The FBA questionnaire was elaborated following a rigorous and consensual protocol, with thorough methodology based on an exploratory analysis of patientreported outcomes, as now recommended by the FDA and $\operatorname{EMEA}^{(17,18)}$.

In view of the psychometric properties of the fortythree item-, eight dimension-version of the FBA questionnaire, the scientific committee decided to delete the pleasure dimension. Its weak test-retest reproducibility over 1 week and its unsatisfactory known-group validity indeed suggested that the two items constituting the pleasure dimension were not stable over time (reproducibility) and that neither could significantly discriminate between individuals differing in their lifestyle and health status (known-group validity). This decision led to a shorter final version of the FBA questionnaire that comprised forty-one items divided into seven dimensions covering vitality, well-being, physical appearance, aesthetics, snacking, disease prevention and digestive comfort.

Overall, the FBA questionnaire presented good psychometric properties. Its reliability was demonstrated, with very good internal consistency reliability and good 


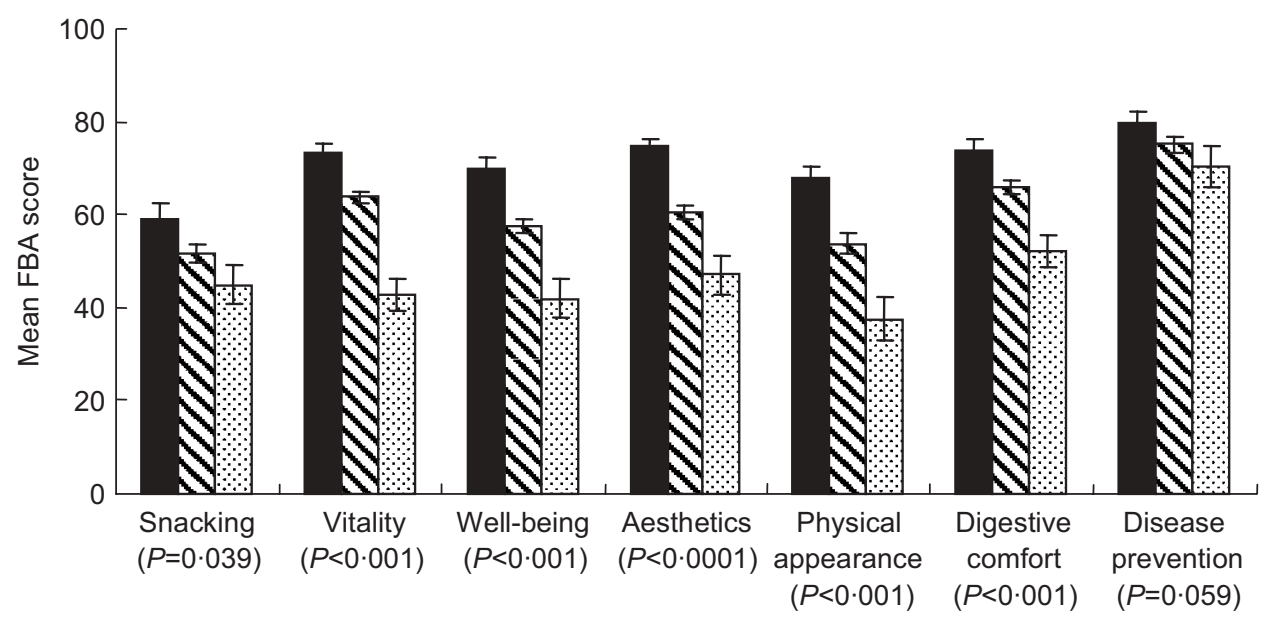

Fig. 4 Comparison between scores of different dimensions of the Food Benefits Assessment (FBA) questionnaire according to subjects' general perception of their health (as defined from their response to the item 'In general, would you say your health is...?' of the Short Form-36 questionnaire): $\mathbf{\square}$, excellent/very good $(n 62) ; \mathbf{\Sigma}$, good $(n 111)$; 2 , fair/poor $(n 20)$. Values are means with their standard deviation represented by vertical bars

test-retest reproducibility over 1 week. The FBA content and construct validity were also evaluated: item convergent and discriminant validity criteria were satisfied; and comparison with the widely used SF-36 questionnaire confirmed the validity of the FBA with the absence of redundancy between the two instruments. Scale-scale correlations showed no overlapping of the FBA concept dimensions. As could have been predicted because of the relative similarity of areas measured by these two concepts, only the vitality and well-being dimensions displayed a higher correlation. Finally, the FBA dimensions proved to be able to discriminate between normal-weight and overweight subjects, as well as subjects with different levels of self-rated general health or presenting different lifestyle habits (i.e. snacking between meals). Subjects' physical appearance and digestive comfort were the domains the most affected by food and diet habits; this was reflected by a high amount of negative and pessimistic answers to questions related to weight and appearance. Altogether, these findings suggest that appearance (including physical appearance and weight) and digestive comfort are very good clues to a good diet/ regimen. In addition, the FBA clearly discriminated between subjects with or without snacking habits between meals. The absence of snacking could be perceived as being related to good food and daily diet by subjects, as people reporting non-snacking had an overall positive attitude and were more satisfied with their diet than people who did snack; they perceived this factor as having positive impacts on their physical and psychological perception of themselves. Interestingly, our sample of normal-weight and overweight subjects, although rather small, followed what one could refer to as 'clichés' or preconceived ideas. Indeed, the majority of overweight subjects reported snacking between meals (64\%) and having more than one drink per week ( $54 \%$ ), while only $36 \%$ and $26 \%$, respectively, of the normal-weight group did so. In addition, subjects within the normal-weight group mostly $(68 \%)$ indicated that they practised at least one sporting activity per week, while only $37 \%$ of subjects in the overweight group did this. One limitation that should be noted is that the majority of the population who participated in the study were women. This could have introduced a bias in the data; especially as it was noted that women were the most aware of the impact of healthy food on disease prevention, with a significantly higher score noticed for women than for men (79 v. 71 , data not shown).

Comparison of the FBA with instruments available in the nutrition field has proved difficult as the latter are essentially devoted to specific digestive and metabolism diseases or disorders ${ }^{(1,14,15)}$, while others are for use with specific populations only ${ }^{(6-8)}$. The concept of a questionnaire allowing the impact of food on HRQoL to be evaluated in the global population is innovative. To our knowledge, only one study reports the development of such an instrument that evaluates the thoughts and feelings about food in the global population ${ }^{(23)}$. The validity and properties of that questionnaire were not studied but, as for the FBA, people demonstrated a genuine interest in participating in such a study ${ }^{(23)}$.

Altogether, these properties make the FBA a helpful instrument that could be administered to the general adult population during randomised controlled trials and observational and epidemiological studies. Its ability to discriminate the effects of a specific food against a control in different domains such as psychological aspects, selfevaluation and physical symptoms would be of great use. In addition, this would facilitate physicians' approaches and arguments when having to prescribe a new or 
modified diet to an individual. According to the Health Belief Model, which states that benefits have to be perceived for someone to adopt a healthy or preventive behaviour $^{(24,25)}$, it is indeed likely that subjects will be more willing to adopt a healthy nutritional behaviour if they can perceive its positive effects and are satisfied with it.

In order to consolidate and further widen its use, future work will consist of determining the FBA's discriminatory properties in other specific population settings such as IBS patients, between two distinct regimens or in an identical regimen but at two different time points. Further studies will allow its validation in an independent population. In any case, the psychometric properties of the FBA will have to be assessed if it is to be included in any new study designs or populations settings. Should future long-term studies be considered, the FBA's sensitivity over time needs to be determined.

\section{Conclusion}

The FBA is the first instrument in the field of nutrition that assesses the impact of food on HRQoL domains, as perceived by subjects. It is a valid and reliable questionnaire, with good psychometric properties, which make the FBA a promising and helpful tool for nutrition health professionals when developing or proposing new products and/or diets.

\section{Acknowledgements}

The work was funded by Danone Research Group. D.G., C.P. and T.L. are employees of Danone Research. O.C., I.G., J.M., E.S., M.V. and E.T. were paid consultants to Danone Research. D.G. participated in the study conception, the development and validation of the questionnaire and in the interpretation of data results; O.C. participated in the development of the questionnaire and in the interpretation of data results; C.P. and T.L. were involved in the initial phase of the questionnaire development and participated in the interpretation of data results; I.G. participated in the manuscript writing and reviewing; J.M. performed the statistical analyses; M.V. participated in the initial step of the questionnaire development; E.S. participated in the validation phase of the questionnaire; E.T. was responsible for methodological directives including conception and design input in the questionnaire development. All authors contributed to the reviewing of the manuscript and agreed with its content.

We would like to thank Professor Roger Jones for his active involvement in the English validation process of the FBA. We also thank Mapi Research Institute (Lyon, France) for the English and German linguistic validations of the questionnaire.
Copyright: The FBA questionnaire is protected by copyright with all rights reserved to Danone Research and must not be used without permission. For information on or permission to use the FBA, please contact Mapi Research Trust, 27 rue de la Villette 69003 Lyon, France. Tel: +33 (0) 4721365 75. Email: trust@mapi.fr. Web site: www.mapi-research.fr.

\section{References}

1. Ahlgren SS, Shultz JA, Massey LK, Hicks BC \& Wysham C (2004) Development of a preliminary diabetes dietary satisfaction and outcomes measure for patients with type 2 diabetes. Qual Life Res 13, 819-832.

2. Hammerlid E, Wirblad B, Sandin C, Mercke C, Edstrom S, Kaasa S, Sullivan M \& Westin T (1998) Malnutrition and food intake in relation to quality of life in head and neck cancer patients. Head Neck 20, 540-548.

3. Pare P, Gray J, Lam S, Balshaw R, Khorasheh S, Barbeau M, Kelly S \& McBurney CR (2006) Health-related quality of life, work productivity, and health care resource utilization of subjects with irritable bowel syndrome: baseline results from LOGIC (Longitudinal Outcomes Study of Gastrointestinal Symptoms in Canada), a naturalistic study. Clin Ther 28, 1726-1735.

4. Ravasco P, Monteiro-Grillo I, Vidal PM \& Camilo ME (2005) Dietary counseling improves patient outcomes: a prospective, randomized, controlled trial in colorectal cancer patients undergoing radiotherapy. J Clin Oncol 23, 1431-1438.

5. Ravasco P, Monteiro-Grillo I, Marques VP \& Camilo ME (2005) Impact of nutrition on outcome: a prospective randomized controlled trial in patients with head and neck cancer undergoing radiotherapy. Head Neck 27, 659-668.

6. Kolotkin RL, Head S, Hamilton M \& Tse CK (1995) Assessing Impact of Weight on Quality of Life. Obes Res 3, 49-56.

7. Kolotkin RL, Crosby RD, Kosloski KD \& Williams GR (2001) Development of a brief measure to assess quality of life in obesity. Obes Res 9, 102-111.

8. Ziegler O, Filipecki J, Girod I \& Guillemin F (2005) Development and validation of a French obesity-specific quality of life questionnaire: Quality of Life, Obesity and Dietetics (QOLOD) rating scale. Diabetes Metab 31, 273-283.

9. Block G, Clifford C, Naughton M, Henderson M \& McAdams M (1989) A brief dietary screen for high fat intake. J Nutr Educ 21, 199-207.

10. Hankin JH, Wilkens LR, Kolonel LN \& Yoshizawa CN (1991) Validation of a quantitative diet history method in Hawaii. Am J Epidemiol 133, 616-628.

11. Guigoz Y \& Vellas BJ (1997) Malnutrition in the elderly: the Mini Nutritional Assessment (MNA). Ther Umsch 54, 345-350.

12. Guigoz Y, Lauque S \& Vellas BJ (2002) Identifying the elderly at risk for malnutrition. The Mini Nutritional Assessment. Clin Geriatr Med 18, 737-757.

13. Keller HH (2004) Nutrition and health-related quality of life in frail older adults. J Nutr Health Aging 8, 245-252.

14. Patrick DL, Drossman DA, Frederick IO, DiCesare J \& Puder KL (1998) Quality of life in persons with irritable bowel syndrome: development and validation of a new measure. Dig Dis Sci 43, 400-411.

15. Reilly WT, Talley NJ, Pemberton JH \& Zinsmeister AR (2000) Validation of a questionnaire to assess fecal incontinence and associated risk factors: Fecal Incontinence Questionnaire. Dis Colon Rectum 43, 146-153. 
16. Chassany O, Sagnier P, Marquis P, Fullerton S \& Aaronson N (2002) Patient-reported outcomes: the example of healthrelated quality of life. A European guidance document for the improved integration of health-related quality of life assessment in the drug regulatory process. Drug InfJ 36, 209-238.

17. Committee for Proprietary Medicinal Product (2007) Points to consider on the evaluation of medicinal products for the treatment of irritable bowel syndrome. http://www.emea. europa.en/pdfs/human/ewp/078597en.pdf (assessed March 2007).

18. Patrick DL, Burke LB, Powers JH, Scott JA, Rock EP, Dawisha S, O'Neil R \& Kennedy DL. (2007) Patient-reported outcomes to support medical product labeling claims: FDA perspective. Value Health 10, Suppl. 2, S125-S137.

19. Olendzki B, Speed C \& Domino FJ (2006) Nutritional assessment and counseling for prevention and treatment of cardiovascular disease. Am Fam Physician 73, 257-264.

20. Acquadro C, Jambon B, Ellis D \& Marquis P (1996) Language and translation issues. In Quality of Life and Pharmacoeconomics in Clinical Trials, 2nd ed., pp. 575-585 [B Spilker, editor]. Philadelphia, PA: LippincottRaven Publishers

21. Ware JEJ, Snow KK, Kosinski M \& Gandek B (1993) SF-36 Health Survey Manual and Interpretation Guide. Boston, MA: New England Medical Center.

22. Hay R \& Hayashi T (1990) Beyond internal consistency reliability: rationale and user's guide for Multitrait analysis program on the microcomputer. Behav Res Methods Instrum Comput 22, 167-175.

23. Rigaud D, Pennacchio H, Van den Berg O, Lalanne Mistrih M-L, Demange K, Huet J-M, Fischler C \& Chiva M (2005) Mise au point d'un questionnaire d'analyse des pensées et sentiments autour de l'alimentation. Cah Nutr Diet $\mathbf{4 0}$, 312-324.

24. Rosenstock IM (1966) Why people use health services. Milbank Mem Fund $Q \mathbf{4 4}$, Suppl. 127.

25. Rosenstock IM (1974) The Health Belief Model and preventive health behavior. Health Educ Monogr 2, 35-86. 